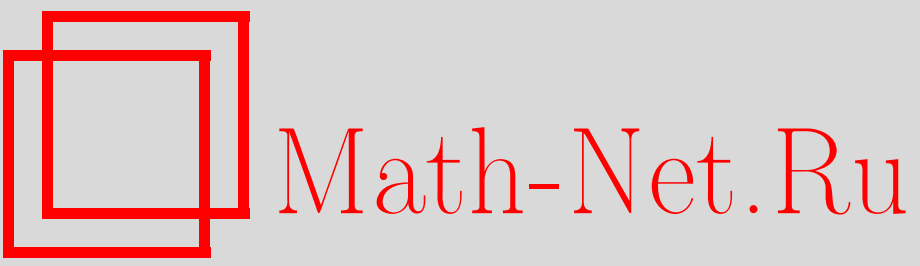

Н. Н. Трунов, Класс потенциалов, для которых квазиклассическое квантование можно сделать точным, ТМФ, 2004, том 138, номер 3, 480-490

DOI: https://doi.org/10.4213/tmf35

Использование Общероссийского математического портала Math-Net.Ru подразумевает, что вы прочитали и согласны с пользовательским соглашением

http://www . mathnet.ru/rus/agreement

Параметры загрузки:

IP : 3.85 .183 .62

26 апреля 2023 г., 14:21:29 
ТЕОРЕТИЧЕСКАЯ

И МАТЕМАТИЧЕСКАЯ

ФИЗИКА

Том 138, № 3

март, 2004

(C) 2004 г.

Н. Н. Трунов*

\section{КЛАСС ПОТЕНЦИАЛОВ, ДЛЯ КОТОРЫХ КВАЗИКЛАССИЧЕСКОЕ КВАНТОВАНИЕ МОЖНО СДЕЛАТЬ ТОЧНЫМ}

Рассмотрен класс потенциалов, для которых достигается точное квазиклассическое квантование посредством определенной модификации условия квантования. Список потенциалов, для которых новое условие точно, совпадает со списком потенциалов, спектр которых определяется методом факторизации. Построено однопараметрическое семейство условий квантования, включающее суперсимметричное ВКБ-условие как частный случай. Новое условие позволяет взаимосвязанно рассмотреть различные модифиикации ведушего приближения и пределы их применимости, а также развить новые приближенные методы вычисления спектров.

Ключевые слова: квазиклассическое приближение, метод факторизации гамильтониана, суперсимметрия в квантовой механике.

\section{1. ВВЕДЕНИЕ}

В последнее время активно предлагаются и изучаются различные новые модификации квазиклассического приближения для определения дискретного спектра. В частности, большой интерес вызывает условие квантования, выраженное через суперпотенциал, а также границы применимости и точность этого условия [1]. Изучается суперсимметричный вариант метода Вентцеля-Крамера-Бриллюэна (ВКБ) и его связь с обычным ВКБ. Делаются попытки расширить список потенциалов, для которых этот вариант точен в первом приближении, строятся контрпримеры, прямым расчетом показывается отсутствие поправок старших порядков для конкретных потенциалов и т.п. [2]. Вводятся различные поправки к стандартной фазе $n+1 / 2$ в правой стороне условия квантования $[3, \S 9.1],[4]$, привлекается квантовый аналог уравнения Гамильтона-Якоби [5], с разных сторон обсуждается и оценивается лангеровская поправка в центральносимметричных задачах [6] и т.д. При этом используются разнообразные приближенные приемы и методы, связь которых между собой, точность и область применимости трудно оценить.

Результаты данной статьи позволяют единообразно рассмотреть значительную часть вышеуказанных вопросов на основе вводимого условия квантования, имеющего

* Всероссийский научно-исследовательский институт метрологии, С.-Петербург, Россия. E-mail: trunov@vniim.ru 
вид первого - основного - приближения, потенциал в котором включает дополнительную "квантовую” добавку. Эта добавка вычисляется на основе второго приближения ВКБ, однако новое условие частично суммирует все члены ряда ВКБ для определения спектра. Показано, что это условие точно для класса потенциалов, спектр которых находится методом факторизации или приведением к уравнению гипергеометрического типа. Оказывается, что учет квантовой добавки лишь перенормирует входяшие в эти потенциалы константы. Таким образом, появляется расширенный (и закрытый) список “эталонных" потенциалов. Квантовая добавка, пропорциональная $\hbar^{2}$, обобщает и обосновывает так называемую лангеровскую поправку. В потенциальных ямах, в которых нижний дискретный уровень сохраняется при сколь угодно малой их глубине, введенная добавка определяет дополнительную фазу $\pi \hbar / 2$ в условии квантования. Показаны преимушества этого модифицированного условия в обшем случае. Построено зависяшее от параметра условие квантования, включающее новое и суперсимметричное условия как частные случаи, и точное для того же класса потенциалов. Показано, что эта же квантовая добавка вместе с номером уровня определяет точность стандартного - ведущего приближения ВКБ.

Полученные соотношения расширяют возможности как обшего анализа, так и приближенных методов, дают практические оценки мере отклонения потенциала от указанного класса, точности ВКБ-приближения для каждого конкретного потенциала, указывают на согласованность условия с дополнительной фазой.

\section{2. УСЛОВИЕ КВАНТОВАНИЯ}

Запишем ряд ВКБ для определения спектра [7] в удобной для дальнейшего форме, сохраняя явный вид первых двух членов разложения по степеням $\hbar^{2}$,

$$
\begin{gathered}
\Phi(E \mid V)+\hbar^{2} R_{2}(E \mid V)-\pi \hbar\left(n+\frac{1}{2}\right)=\hbar^{4} Q(E \mid V), \\
\Phi(E \mid V)=\int \sqrt{2(E-V(x))} d x \\
R_{2}(E \mid V)=-\frac{1}{24} \frac{\partial^{2}}{\partial E^{2}} I, \quad I=\int \frac{d x}{\sqrt{2(E-V(x))}}\left(\frac{\partial V}{\partial x}\right)^{2} .
\end{gathered}
$$

Через

$$
Q=\sum_{n=2}^{\infty} \hbar^{2(n-2)} R_{2 n}
$$

обозначен весь остаток ряда. Интегралы с необозначенными пределами берутся между точками поворота. Здесь и далее мы полагаем массу $m=1$. Потенциалы предполагаются аналитическими функциями в некоторой области на комплексной плоскости, охватываюшей отрезок вешественной оси между точками поворота (т.е. как функции $x$ они аналитичны в классически доступной области).

Ниже рассматриваются только потенциалы, имеющие один параболический минимум $v \equiv V_{\min }=V\left(x_{\min }\right)$ с монотонными склонами потенциальной ямы. Для потенциалов кулоновского типа эти условия обеспечиваются введением центробежного члена

5 Теоретическая и математическая физика, т. 138, № 3, 2004 г. 
(который в конце вычислений может быть устремлен к нулю). Для таких потенциалов можно перейти к переменной $V$, введя на каждом склоне положительные функции $g$ и $f$, согласованные при $V \rightarrow v: g(V)=d V / d x, x \geqslant x_{\min }, f(V)=-d V / d x, x \leqslant x_{\min }$,

$$
\begin{aligned}
& g(V)=C(V-v)^{1 / 2}+D(V-v)+E_{+}(V-v)^{3 / 2}+\cdots \\
& f(V)=C(V-v)^{1 / 2}-D(V-v)+E_{-}(V-v)^{3 / 2}+\cdots
\end{aligned}
$$

Обратим внимание на совпадение коэффициентов $C$ и (с точностью до знака) $D$ в разложении функций $g$ и $f$. Условие (1) принимает вид

$$
\begin{gathered}
\Phi[g]+\Phi[f]+\hbar^{2}\left(R_{2}[g]+R_{2}[f]\right)-\pi\left(n+\frac{1}{2}\right) \hbar=\hbar^{4} Q \\
\Phi[g]=\int_{v}^{E} \sqrt{2(E-V)} \frac{d V}{g(V)}, \quad R_{2}[g]=-\frac{1}{24} \frac{\partial^{2}}{\partial E^{2}} \int_{v}^{E} \frac{d V g(V)}{\sqrt{2(E-V)}}
\end{gathered}
$$

так что $\Phi(E \mid V)=\Phi[g]+\Phi[f]$ и $R_{2}(E \mid V)=R_{2}[g]+R_{2}[f]$.

УТВЕРЖДЕНИЕ 2.1. Пара функиий $\Phi(E \mid V)$ u $R_{2}(E \mid V)$ из (2), (3) находятся во взаимно однозначном соответствии с потенциалом $V(x)$ указанного типа.

Здесь и ниже для доказательства используем свойства уравнения Абеля

$$
z(\tau)=\int_{a}^{\tau} \frac{y(t) d t}{\sqrt{\tau-t}}, \quad \pi y(\tau)=\frac{d}{d \tau} \int_{a}^{\tau} \frac{z(t) d t}{\sqrt{\tau-t}}=\int_{a}^{\tau} \frac{z^{\prime}(t) d t}{\sqrt{\tau-t}}
$$

причем последнее равенство в (7) справедливо при $z(a)=0$ (фактически мы имеем дело с оператором интегрирования дробного порядка $1 / 2)$. Решение (7) единственно в классе непрерывно дифференцируемых функций $z(\tau)$.

Выразим производную $\Phi$ в уравнении (1) по энергии через потенциал $V$. Тогда, как видно из $(6)$, в интеграл войдет $1 / g+1 / f$. Аналогично $R_{2}$ будет включать $g+f$. Пользуясь выражениями (7), получим уравнения

$$
\begin{gathered}
\frac{1}{g}+\frac{1}{f}=\frac{2}{\pi} \frac{d}{d V} \int_{v}^{V} \frac{d \mathcal{E}}{\sqrt{V-\mathcal{E}}} \frac{d \Phi}{d \mathcal{E}} \\
\pi \frac{d}{d V}(g+f)=\int_{v}^{V} \frac{R_{2}(\mathcal{E}) d \mathcal{E}}{\sqrt{V-\mathcal{E}}}
\end{gathered}
$$

определяюшие каждую из функций $g, f$ с учетом (4), а с ними и потенщиал $V(x)$. Обратно, из соотношений $(8)$ с учетом условия $\Phi(E=v)=0$ функции $\Phi$ и $R_{2}$ находятся по функциям $g$ и $f$.

Старшие члены ряда теории ВКБ являются функционалами $V$, так что они полностью определяются парой $\left(\Phi, R_{2}\right)$. Напомним, что одна функция $\Phi$ определяет лишь некоторый класс эквивалентности потенциалов со спектрами, совпадающими в первом приближении.

Для последующего анализа целесообразно избавиться в $R_{2}$ из соотношения (3) от дифференцирования интеграла. 
УТВЕРЖДЕнИЕ 2.2. Для рассматриваемых потенциалов функция $R_{2}$ из (6) представима в виде

$$
R_{2}[g]=-\int_{v}^{V} \frac{d V \tilde{r}[g]}{\sqrt{E-V}}=-\int_{x_{l}}^{x_{\min }} \frac{d x r(x)}{\sqrt{E-V(x)}}
$$

ฉде

$$
\tilde{r}[g]=\frac{1}{24} \frac{d^{2} G}{d V^{2}}, \quad G=g(V)-C(V-v)^{1 / 2}, \quad r(x)=g \tilde{r}[g],
$$

$x_{l}<x_{\min }$ - левая точка поворота, а коэффициент $C$ из (4) и функция $r(x)$ возникают после подстановки в $\tilde{r}[g(V)]$ и $g(V)$ значения $V(x)$.

Заметим, что заменой $d / d V=g^{-1}(d / d x)$ можно привести $r(x)$ к виду, содержашему только производные от $V$ по $x$ (не по $V$ ).

Добавление в интеграл $I(3)$ члена с $\sqrt{V-v}$ не меняет значения $R_{2}$, так как линейная по $E$ добавка к $I$ исчезает при двукратном дифференцировании. В то же время выражение $R_{2}(6)$ с функцией $G(9)$ вместо $g$ допускает, как в (7), внесение производной под знак интеграла, так как $d G /\left.d V\right|_{v}=0$. Аналогичное выражение для $R_{2}[f]$ получим заменой $g \rightarrow f$; оно соответствует интервалу $\left(x_{\min }, \infty\right)$. Заметим, что $\tilde{r}[g]$ и $\tilde{r}[f]$ не зависят от линейных членов в (4), что находится в соответствии с возможностью замены в функционалах $R_{2}[g], R_{2}[f]$ из (5) функций $g$ и $f$ на $g+\widetilde{D} V, f-\widetilde{D} V$ с сохранением суммы этих функционалов, т.е. $R_{2}$. Напомним, что члены ряда теории возмущений первоначально представляются контурными интегралами, так что подынтегральная функция определяется с точностью до полной производной. Для перехода к интегрированию между точками поворота необходимо предварительно все знаменатели вида $(E-V)^{-k} \mathrm{c}$ $k>1 / 2$ выразить через производные по $E$, как в $R_{2}(3) ;$ далее мы не оговариваем этого специально.

\section{3. ВЫБОР УСЛОВИЯ КВАНТОВАНИЯ ИЗ КЛАССА ЭКВИВАЛЕНТНОСТИ}

Список потенциалов, для которых точньй спектр получается в ведушем приближении ВКБ (когда $R_{2}=Q \equiv 0$ в (1)) крайне узок: осциллятор и потенциал Морса (для них $\left.R_{2} \equiv 0\right)$. Этот список не расширяется учетом второго приближения, т.е. учетом $R_{2} \neq 0$ при $Q \equiv 0$. Более того, известны потенциалы, для которых точный спектр дает несколько измененное условие в ведущем приближении, выраженное через суперпотенциал (см. ниже раздел 6); легко проверить, что для таких потенциалов $R_{2 n} \neq 0$ при всех $n \geqslant 1$.

Целесообразно поэтому перестроить ряд (1) так, чтобы максимально расширить список потенциалов, точный спектр которых давался бы новым ведушим приближением. Оно явилось бы основой эффективного приближенного расчета для других потенциалов.

Осуществив в функционале $\Phi$ в формуле (1) замену $V \rightarrow V+\hbar^{2} u$ с достаточно гладкой функцией $u$, имеюшей то же поведение, что и $V$, и одновременно вычитая ряд теории возмушений по $\hbar^{2} u$, получим вместо (1) уравнение

$$
\begin{gathered}
\Phi\left(E \mid V+\hbar^{2} u\right)+\hbar^{2}\left(R_{2}(E \mid V)+\int \frac{u(x) d x}{\sqrt{2(E-V(x))}}\right)-\pi \hbar\left(n+\frac{1}{2}\right)= \\
=\hbar^{4}(U(E \mid u, V)+\widetilde{Q}(E \mid u, V)) \equiv \hbar^{4} W(E \mid u, V) .
\end{gathered}
$$


Здесь $U$ - остаток ряда разложения $\Phi$ по степеням $\hbar^{2} u$ (первый член выписан явно), а $\widetilde{Q}$ - остаток ряда $Q$ после замены $V$. Заметим, что функция $R_{2}$ (и только $R_{2}$ ) не зависит от $u$.

Обычный ряд имеет $u \equiv 0$. Распорядимся произволом в выборе функции $u$. Естественно потребовать обрашения в нуль членов порядка $\hbar^{2}$ в $(10)$. В соответствии с $(6),(9)$ это приводит к условию $u(x)=r(x)$, где функция $r(x)$ определена в $(9)$.

УТВЕРЖДЕНИЕ 3.1. Функиия $r(x)$ зависит от формы, но не от амплитуды потенциала, т.е. инвариантна при замене $V \rightarrow A V$ с постоянной $A>0$.

Это следует непосредственно из того, что

$$
r(x) \sim \frac{\partial V}{\partial x} \frac{\partial^{2}}{\partial V^{2}} \frac{\partial V}{\partial x} \sim A^{0} .
$$

Для естественного учета этой инвариантности удобно ввести вспомогательную монотонную функцию $s(x)$ такую, что

$$
V-v=s^{2}(x), \quad \sigma=\frac{d s}{d x}>0 .
$$

Функцию $s$ можно трактовать как искажение профиля потенциала гармонического осциллятора, для которого $s(x)=k x$. Выражая $r$ из (9) через $s$ и $\sigma$, получим

$$
r(s)=\frac{\sigma}{24} \frac{d}{d s} \frac{1}{s} \frac{d}{d s}(\sigma(s)-\sigma(0)) s .
$$

Для данного $V(x)$ функция $r$ может иметь сложный вид. Как увидим ниже, представляют интерес простейшие виды $r$, для которых справедливы утверждения, непосредственно проверяемые с помощью формулы (11).

УТВЕРЖДЕНИЕ 3.2. Квантовая поправка $r$ или равна нулю, или линейна по $V$, т.е. имеет вид $\alpha s^{2}+\beta$, соответственно при

$$
\sigma(s)=a_{0}+a_{1} s, \quad r \equiv 0,
$$

$u$

$$
\sigma(s)=a_{2} s^{2}+a_{0}, \quad r=\frac{1}{8} a_{2}\left(a_{2} s^{2}+a_{0}\right) .
$$

УТВЕРЖДЕНИЕ 3.3. Квантовая поправка сохраняет свой вид (13) для потенциалов с произвольными значениями коэффициентов

$$
V=\sum_{k=0}^{2} b_{k} s^{k}, \quad \sigma(s)=\sum_{k=0}^{2} a_{k} s^{k} .
$$

Таким образом, в классе потенциалов (14) учет второго приближения только перенормирует константы $b_{2}, b_{0}$ в $V$ (но не константу $b_{1}$, которая в кулоновском потенциале пропорциональна заряду). Класс потенциалов (14) инвариантен относительно замен $s \rightarrow d_{1} s+d_{0}$; при этом нарушается вид $\sigma$ из (13) - появляется линейный по $s$ член, однако вид $r$ (13) сохранится. Целесообразно выбирать $\sigma(s)$ в виде $(12)$ или (13), т.е. с $a_{2} a_{1}=0$, в последнем случае подбором $d_{0}$. Как видно из $(13)$, представление потенциалов в форме (14) приводит к зависимости $r$ только от $\sigma$, но не от $V$, в соответствии с утверждением 3.1 . 
УТВЕРЖДЕНИЕ 3.4. В условии (5) $R_{2}[g], \quad R_{2}[f]$ не зависят от $Е$ для (14) и только для (14), причем $R_{2} \equiv 0$ при выполнении условия (12).

Заметим, что $R_{2}=R_{2}[g]+R_{2}[f]$ можно рассматривать как поправку $\gamma^{\prime}=-\hbar R_{2} / \pi$ к фазе $1 / 2$ в (1).

\section{4. СВОЙСТВА НОВОГО УСЛОВИЯ}

Потенциалы (14) оказываются выделенными еше в одном, весьма важном отношении.

УТВЕРЖДЕНИЕ 4.1. Условие квантования

$$
\int \sqrt{2\left(E-V(x)-\hbar^{2} r(x)\right)} d x=\pi \hbar\left(n+\frac{1}{2}\right)
$$

является точным для всех потенциалов (14) при всех значениях входящих в них параметров $\left(a_{k}, b_{k}\right)$.

Доказать это можно разными способами.

Во-первых, проследив за структурой дальнейших членов ряда теории возмушений, убедиться, что $W \equiv 0$. Например, для $\sigma(12)$, вычисляя $R_{2 n}$, приведенное в [7], с переходом к $s, V(s)$ и $\sigma$, легко убедиться, что все $R_{2 n} \equiv 0(n \geqslant 1)$, и условие (15) точно.

Во-вторых, достаточно заметить, что уравнение Шредингера, выраженное через $s$ с $V$ и $\sigma(14)$, является уравнением гипергеометрического типа $[8, \S 1,2]$ с элементарно определяемым спектром. Сравнение его с получаемым по (15) для разных вариантов $\sigma$ $(12),(13)$ обнаруживает их совпадение (с учетом для отдельных потенциалов преобразования симметрии $[8$, c. 321]). Получаемьй при этом список потеншиалов точно совпадает с найденным в [9] на основе значительно более сложного и громоздкого анализа различных вариантов факторизации уравнения Шредингера. Замены переменных "ad hoc" в [9] и других работах автоматически учитываются в форме (14). Фактически при этом систематизируется и исчерпьвается список потеншиалов, для спектра которых получены точные выражения (не через корни спецфункций $)^{1}$. Эти потенциалы имеют разное качественное поведение и асимптотики, спектр их имеет один из видов

$$
\text { a) } E_{n}=P_{1}(n), \quad \text { б) } E_{n}=\sum_{k=-1}^{1} c_{k} P_{1}^{2 k}(n)
$$

где $P_{1}$ - полином первой степени; здесь достигаются предельно возможные степени $E_{n} \sim$ $n^{2}, E_{n} \sim-n^{-2}$.

В-третьих, можно, что для нас содержательнее, связать наше условие (15) с суперсимметричным; основанное на этом доказательство приведено ниже в разделе 6 .

В классе (14) зависимость всех $R_{2 n}$ от $R_{2}$ видна явно: в каждом порядке по $\hbar^{2}$ член ряда теории возмушений по $\hbar^{2} u$ ( $u$ определяется через $R_{2}$ ) компенсирует $R_{2 n}$ в $W(10)$.

Наличие целого списка качественно различных потенциалов, для которых условие (15) точно независимо от выполнения известных условий квазиклассичности $[10, \S 46]$,

\footnotetext{
1) Заметим, что фактически именно потенциалы из этого списка используются для тестирования вводимых "ad hoc" поправок к стандартному ВКБ.
} 
$[3, \S 9.1]$, во всем диапазоне параметров вплоть до $b_{k}=0$ и при всех $n \geqslant 0$ (так что оба слагаемых под знаком радикала в (15) равноправны, а разложение $(10)$ - лишш способ расчета $r$, приводит к иной точке зрения на метод вычисления спектра по условию (15). Именно, для потенциала обшего вида погрешность условия (15) имеет порядок $O\left(\hbar^{4}\right)$. Однако величина $W$, тождественно равная нулю для указанного списка, будет мала для потенциалов, близких (по некоторому расстоянию в функциональном пространстве потенциалов) к (14). Поскольку список (14) включает разнотипные потенциалы с широким диапазоном параметров, можно рассчитывать на близость реальных потенциалов к эталонным (14), т.е. на малость $W$. Практическим критерием близости может служить величина первого члена ряда $U+\widetilde{Q}$ в $(10)$ или, по-другому, отличие вида функции $r$ от эталонного.

Точность условия (15) для целого класса потенциалов неявно означает его повышенную симметрию, что выделяет (15) как оптимальный с групповой точки зрения $[11$, с. 9$]$ вариант условия (в обшем случае, приближенного).

Заметим, наконец, что переход к (15) от (1) означает частичное суммирование членов ряда всех степеней по $\hbar^{2} r$ ("ведущих поправок"; в классе (14) - полное суммирование).

Независимость $V_{q}=\hbar^{2} r$ в (15) от энергии позволяет считать $V_{q}$ квантовой добавкой к потенциалу $V$, имеющей самостоятельное значение, например, при квантово-механических расчетах с использованием уравнения Гамильтона-Якоби [5]; радикал в (15) представляет удобную форму вводимого там “квантового импульса". Эффективный потенциал, включаюший зависимость от $\hbar$, возникает и в других задачах [12], [13]. Наша форма $V_{q}$ отличается, в частности, определенным - вторым - порядком по $\hbar$. Член порядка $\hbar^{2}$ иногда включают в фазу ВКБ волновой функции $[14$, с. 44$]$, он зависит от энергии и, разумеется, не совпадает с $V_{q}$.

\section{5. МОДИФИКАЦИЯ ВКБ-УСЛОВИЙ КВАНТОВАНИЯ НА ОСНОВЕ НОВОГО УСЛОВИЯ}

Как известно, для уточнения спектра, получаемого из ВКБ-условия в ведущем приближении (т.е. (1) с $\left.R_{2}=Q \equiv 0\right)$, применяют модификации этого условия, основанные на различных приемах, точность и область применимости которых обычно неясны. Условие (15) позволяет прояснить многие возникающие здесь вопросы практически без вычислений.

Начнем с лангеровской добавки для центрально-симметричных задач: постулируется, что в условии (1) должен быть изменен коэффициент в центробежном потенциале, а именно $\hbar^{2} l(l+1) /\left(2 x^{2}\right)$ заменяется на $\hbar^{2}(l+1 / 2)^{2} /\left(2 x^{2}\right)$, где $l$ - орбитальный момент $[10, \S 49]$.

Сравним два потенциала из класса (14) - кулоновский и хюльтеновский,

$$
\begin{gathered}
w=x^{-1}=-s, \quad \sigma=s^{2}, \\
w=\left(e^{x}-1\right)^{-1}=\frac{1}{2}-s, \quad s=-\frac{1}{2} \operatorname{cth} \frac{x}{2}, \quad \sigma=s^{2}-\frac{1}{4} .
\end{gathered}
$$

Для образования минимума $V$ при $x \neq 0$ можно ввести квадратичный по $w$ член с произвольным коэффициентом $L>0$, устремляемым к нулю в конше расчетов, т.е

$$
V=L w^{2}-w .
$$


Точньй спектр для потенциалов (17), (16) дает условие (15) с учетом члена $\hbar^{2} r$, причем соответственно (16) имеем
a) $r=\frac{w^{2}}{8}$
б) $r=\frac{w(w+1)}{8}$

Таким образом, в обоих случаях перенормируется коэффициент $L: L \rightarrow L+\hbar^{2} / 8$, а в случае "б" появляется и добавка, линейная по $w$, если рассматривать (15) как обычное условие в ведушем порядке. В частности, при $L=\hbar^{2} l(l+1) / 2$ получаем замену $l(l+1) \rightarrow(l+1 / 2)^{2}$, однако только для кулоновского случая слагаемое с $w^{2}$ в $(17)$ действительно является центробежным потенциалом. В случае же (16б) появляется возмушение $L\left(w^{2}-x^{-2}\right)$, нарушающее точность определения спектра при всех $l$, кроме $l=L=0$. Таким образом, стандартная лангеровская замена не является универсальной даже в классе $(14)^{2)}$. Иными словами, обобщением лангеровской поправки на любые потенциалы является именно $\hbar^{2} r$.

Другое направление модификации условия квантования связано с изменением правой стороны этого условия в форме (15). Сюда относится, например, исключение из $\Phi$ центробежного потенциала с добавлением в правую сторону условия к $n+1 / 2$ члена $l+1 / 2[3$, с. 460]. Такие замены в классе (14) выполняются так же, как изменение параметра в разделе 6 (см. ниже); к ним относятся все ограничения точности, указанные для лангеровской поправки.

Отдельно упомянем потенциалы с одинаковыми асимптотическими значениями; для определенности будем считать, что

$$
V(x \rightarrow \infty)=V(x \rightarrow-\infty)=0 .
$$

Такой случай актуален при анализе появления новых уровней в атомах [16]. Запишем потенциал $V(x)<0$ в виде $V=A^{2} w(x), \max _{x}|w(x)|=1$. Условие (15) примет вид

$$
\int \sqrt{2\left(E-A^{2} w(x)-\hbar^{2} r(x)\right)} d x=\pi \hbar(n+\gamma), \quad \gamma=\frac{1}{2},
$$

функция $r(x)$ не зависит от амплитуды $A$. Как известно [18, с. 22], в потенциальных ямах с условием (18) один нижний уровень с $n=0$ существует даже при $A \rightarrow 0$, тогда и $|E| \sim$ $A^{4} \rightarrow 0$. Равенство (19) в этом пределе имеет одинаковый, первый порядок по $\hbar$ в обеих сторонах. При этом выполняется условие согласования

$$
\sqrt{2} \int \sqrt{-r(x)} d x=\pi \gamma
$$

Так, при $w=-\operatorname{ch}^{-2} x$ имеем (см. раздел 3$): v=-A^{2}, s=A$ th $x$. Из (13) получим

$$
\sigma=\frac{d s}{d x}=\frac{A^{2}-s^{2}}{A}, \quad r=-\frac{\sigma}{8 A}=\frac{w}{8} .
$$

\footnotetext{
${ }^{2)}$ Вместе с тем лангеровское преобразование координат $y=\ln x$, как выделенное по симметрии [15], сохраняет свое значение для развития приближенных методов расчета спектра и для введения эффективного квантового числа в центрально-симметричных задачах [16], [17].
} 
Подстановка $r$ в интеграл (20) (по всей оси) дает в левой стороне $\pi / 2$. Таким образом, равенство (20) действительно вьполняется при стандартном значении $\gamma=1 / 2$ (справедливом для экспоненциально убьвающих потенциалов при всех $E$ вплоть до $E=0$ ).

При всех значениях $A$ вычисление интеграла (19) приводит, как следует из предыдушего, к точному спектру $[10$, с. 100$]$.

За пределами класса (14) вычисление $r$ может привести к другому значению $\gamma \neq 1 / 2$, что позволит подправить правую часть условия (например, известно, что для медленно убываюшего потенциала $w=-\left(x^{2}+1\right)^{-2}$ в момент появления уровня $E=0$ точно ведушее приближение ВКБ с $\gamma=1$ ).

В заключение раздела на примере потенциалов (18) покажем, как $r$ определяет точность ведущего приближения ВКБ. Проследим за появлением $N$-го, $N=n+1$, уровня (с энергией $E=0)$. Из разложения $(19)$ найдем с точностью $O\left(\hbar^{4}\right)$

$$
A \int \sqrt{-2 w(x)} d x+\frac{\hbar^{2}}{24 A} \int \frac{r(x) d x}{\sqrt{-2 w(x)}}=\left(N-\frac{1}{2}\right) \pi \hbar .
$$

Таким образом, $\hbar^{2}$ появляется в условии всегда в комбинации $(\hbar / A)^{2}$, что делает разложение некорректным при $A \rightarrow 0$. Относительная погрешность, возникаюшая при игнорировании $r(x)$, есть

$$
\delta=\frac{\bar{r}}{24 \pi^{2}\left(N-\frac{1}{2}\right)^{2}}, \quad \bar{r}=\int \sqrt{-w(x)} d x \int \frac{r(x) d x}{\sqrt{-w(x)}} .
$$

Отсюда ясно, что точность ведушего приближения ВКБ определяется не номером уровня или видом потенциала (а с ним и $r$ ) в отдельности, а именно их комбинацией. В частности, при $r \equiv 0$ первое приближение точно при всех $N \geqslant 1$. Заметим также, что отношение $\hbar / A \sim N^{-1}$, так что разложение по $(\hbar / A)^{2}$ в действительности идет по параметру $N^{-2}$. Напомним, что точность условия (15) (или (19)) определяется не значением $r$, а отличием функции $r$ или $R_{2}$ от соответствуюшей классу (14) (см. утверждение 3.4 ).

\section{6. ПАРАМЕТРИЧЕСКОЕ СЕМЕЙСТВО УСЛОВИЙ КВАНТОВАНИЯ}

Известно [1], что для ряда потенциалов специального вида модифицированное условие квантования при выражении потенциала $V$ через суперпотенциал $S$ в соответствии с формулами

$$
\int \sqrt{2\left(E-S^{2}(x)\right)} d x=\pi \hbar n, \quad V_{ \pm}=S^{2} \pm \frac{\hbar}{\sqrt{2}} \frac{d S}{d x}
$$

дает точные выражения для спектра. Здесь имеется два отличия от обычного условия в ведушем приближении: $S^{2}$ вместо $V$ под интегралом и $n$ вместо $n+1 / 2$ в правой стороне. Напомним, что проявлением суперсимметрии в квантовой механике является совпадение спектров для пары потенциалов $V_{ \pm}$, именно $E_{n}^{-}=E_{n-1}^{+}$при $n \geqslant 1$.

Очевидно, при $\sigma$ из (14) потенциалы $V_{ \pm}$являются частным случаем $V(14)$. Для изучения взаимосвязи условий (15) и (21), каждое из которых в ряде случаев приводит к точному выражению для спектра, введем двухпараметрическое семейство

$$
\Pi(\alpha, \beta, x)=s^{2}+\frac{\alpha \hbar}{\sqrt{2}} \sigma+\beta \hbar^{2} r
$$


где $r$ определено формулой (11). Это семейство включает как частные случаи $V_{ \pm}(21)$ при $\alpha= \pm 1, \beta=0$, потенциалы вида (14) с $b_{1}=b_{0}=0$ при $\alpha=0, \beta=0$ и тот же потенциал с квантовой добавкой при $\alpha=0, \beta=1$.

УТВЕРЖДЕНИЕ 6.1. Допустим, что для данной функиии б (14) точно одно из равенств: (15) для $V_{ \pm}$или (21). Тогда при $a_{2} a_{1}=0$ для всех значений $\alpha$ (обеспечивающих наличие данного связанного состояния) при $\beta=\alpha^{2}$ имеет место соотношение

$$
\Phi(\alpha)=\int \sqrt{2\left(E-\Pi\left(\alpha, \alpha^{2}, x\right)\right)} d x=\pi \hbar\left(n+\frac{\alpha}{2}\right) .
$$

При $\alpha=0$, очевидно, выражение (23) совпадает с (21), а при $\alpha= \pm 1$ оно совпадает c (15) для потенциала $V_{ \pm}$. Поэтому достаточно показать, что $d \Phi(\alpha) / d \alpha=\pi \hbar / 2$. Переходя в $(23)$ к интегрированию по $d s=d x / \sigma$, получим с учетом $r=a_{2} \sigma / 8$ для $d \Phi / d \alpha$ интеграл вида

$$
\int_{B}^{A} \frac{d s}{\sqrt{(A-s)(s-B)}}=\pi,
$$

не зависящий от $A, B$ (в данном случае $B=-A$ ). Это приводит к $(23)$, а также к следуюшему утверждению.

УТВЕРЖДЕНИЕ 6.2. В случае $\sigma$ общего вида (14) с $a_{1} a_{2} \neq 0$ равенство (23) сохраняется после выполнения в П (22) сдвига s, обеспечивающего отсутствие линейного члена в $\sigma$,

$$
s \rightarrow s-\frac{a_{1}}{2 a_{2}}
$$

c соответственным изменением $r$.

Отметим, что при $\alpha=0$, когда квантовая добавка не фигурирует в (23), условия (21) и (23) переходят друг в друга путем сдвига $s$ при любой форме $\sigma(14)$. При $\alpha \neq 0$ этому препятствует указанная выше некоммутативность операций сдвига и вычисления квантовой поправки (см. раздел 3$)$.

Учитывая точность условия (10) в классе потенциалов (14) и выделяя в П $\left(\alpha, \alpha^{2}, x\right)$ квантовую поправку, придем к утверждению, следуюшему из (23).

УТВЕРЖДЕНИЕ 6.3. Семейство потенииалов

$$
V(\alpha, x)=s^{2}+\frac{\alpha \hbar}{\sqrt{2}} \sigma+\left(\alpha^{2}-1\right) \hbar^{2} r
$$

где б имеет вид (14) при $a_{1} a_{2}=0$, а при $a_{1} a_{2} \neq 0$ получаемое заменой (24) в (25), обладает подобными спектрами со сдвиговой симметрией:

$$
E_{n}(\alpha)=E_{n+\gamma}(1), \quad \gamma=\frac{\alpha-1}{2} .
$$

Здесь Е рассматривается как функиия непрерывной переменной $n$.

Очевидно, формулы (25), (26) обобщают связь спектров суперсимметричной пары при $\alpha= \pm 1$ (21) на произвольные $\alpha$, но с нулевой погрешностью только для потенциалов (14) (при $\alpha= \pm 1$ потенциал (25) совпадает с (21) для всех $V_{ \pm}$).

Вернемся к условию (23) с потенциалом П $\left(\alpha, \alpha^{2}, x\right)$. Из непрерывной по $\alpha$ связи условий (21) при $\alpha=0$ и (15) при $\alpha=1$ и потенциалах (14) следует, что эти условия рав- 
носильны в пределах класса (14). Выполнив указанную в разделе 4 проверку точности условия (15) для потенциалов (14), получим, что спектры всех потенциалов (14) удовлетворяют и суперсимметричному уловию $(21)$, причем все $V_{ \pm}$приводят к уравнению Шредингера гипергеометрического типа.

Наоборот, воспользовавшись полученным независимой проверкой утверждением [1], что условие (21) точно для форм-инвариантных потенциалов с адлитивным параметром и список их совпадает с [9], придем к утверждению 4.1.

Полученные выше результаты приводят к следуюшим основным выводам. Условие $(15)$, обладаюшее малой погрешностью $O\left(\hbar^{4}\right)$ для потенциалов обшего вида, является точным в классе потенциалов, спектр которых может быть получен алгебраически. Тем самым оно связывает и объединяет функциональный (ВКБ) и дискретно-математический подходы. Так, например, результаты вычисления спектров методом факторизации [19] могут быть реинтерпретированы как квазиклассические согласно (15). Учитывая (15), можно приближенно распространить алгебраический подход на более широкий круг потенциалов.

Благодарности. Автор благодарит А. А. Лобашева за полезные обсуждения и содействие при подготовке статьи к печати.

\section{Список литературы}

[1] F. Cooper, A. Khare, U. Sukhatme. Phys. Rep. 1995. V. 251. P. 267; hep-th/9405029.

[2] M. Hrŭska, W. Y. Keung, U. Sukhatme. Phys. Rev. A. 1997. V. 55. P. 3345.

[3] В. М. Галичкий, Б. М. Карнаков, В. И. Коган. Задачи по квантовой механике. М.: Наука, 1992.

[4] M. Friedrich, J. Trost. Phys. Rev. A. 1996. V. 54. P. 1136.

[5] R. A. Leacock, M. J. Padgett. Phys. Rev. D. 1983. V. 28. P. 2491.

[6] J. Hainz, H. Grabert. Phys. Rev. A. 1999. V. 60. P. 1698.

[7] С. Ю. Славянов. Асимптотика решений одномерного уравнения Шредингера. Л.: Изд-во ЛГУ, 1990.

[8] А.Ф. Никифоров, В. Б. Уваров. Специальные функции математической физики. М.: Наука, 1984.

[9] L. Infeld, T. E. Hull. Rev. Mod. Phys. 1951. V. 32. № 1. P. 21.

[10] Л. Д. Ландау, Е. М. Лифииц. Квантовая механика. Теоретическая физика. Т. 3. М.: Наука, 1988.

[11] Л. В. Овсянников. Групповой анализ дифференциальных уравнений. М.: Наука, 1978.

[12] А. Д. Суханов. ТМФ. 2002. Т. 132. № 3. С. 449.

[13] P. Holland. The Quantum Theory of Motion. Cambridge: Cambridge Univ. Press, 1993.

[14] М. В. Федорюк. Асимптотические методы для линейных обыкновенных дифференциальных уравнений. М.: Наука, 1983.

[15] К. С. Мамаева, Н. Н. Трунов. ТМФ. 2003. Т. 135. № 1. С. 82.

[16] А. А. Лобашев, Н. Н. Трунов. ТМФ. 1999. Т. 120. № 1. С. 99; 2000. Т. 124. № 3. С. 463.

[17] Yu. V. Tarbeyev, N. N. Trunov, A.A. Lobashev, V.V. Kukhar. Effective quantum number of centrally symmetric potentials. In: Operator Methods in Ordinary and Partial Differential Equations. Proc. of the Sonja Kovalevsky Symp. (Stockholm, June 16-22, 2000). Operator Theory: Advances and Applications. V. 132. Eds. S. Albeverio, N. Elander, W. N. Everitt, P. Kurasov. Basel: Birkhäuser, 2002. P. 403.

[18] А.И. Базь, Я. Б. Зельдович, А. М. Переломов. Рассеяние, реакции и распады в нерелятивистской квантовой механике. М.: Наука, 1971.

[19] Х. Грин. Матричная квантовая механика. М.: Мир, 1968.

Поступила в редакцию 10.II.2003 г., после доработки 3.IV.2003 г. 